\title{
Obstructive sleep apnoea and coronary artery disease
}

\author{
Swapna Mandal ${ }^{1,2}$, Brian D. Kent ${ }^{3,4}$ \\ ${ }^{1}$ Department of Respiratory and Sleep Medicine, Royal Free Hospital, London, UK; ${ }^{2}$ University College London, UK; ${ }^{3}$ Sleep Disorders Centre, \\ Guy's \& St. Thomas' Hospitals, London, UK; ${ }^{4}$ King's College London, London, UK \\ Contributions: (I) Conception and design: All authors; (II) Administrative support: None; (III) Provision of study materials or patients: None; \\ (IV) Collection and assembly of data: All authors; (V) Data analysis and interpretation: All authors; (VI) Manuscript writing: All authors; (VII) Final \\ approval of manuscript: All authors. \\ Correspondence to: Brian D. Kent. Sleep Disorders Centre, Guy’s \& St. Thomas’ Hospitals, London SE1 9RT, UK. Email: brian.kent@kcl.ac.uk.
}

\begin{abstract}
Obstructive sleep apnoea (OSA) is a highly prevalent disorder, which causes significant nocturnal and daytime symptoms, reduced quality of life, and impaired functional capacity. Importantly, however, OSA also appears to predispose to the development of a number of cardiovascular and metabolic diseases, including diabetes, hypertension, and stroke. In this review we explore its relationship with coronary artery disease (CAD), discussing mechanisms whereby it may promote the development of atherosclerosis, evidence of its effect on CAD incidence and outcomes, and coronary imaging studies in subjects with OSA. Finally, we shall evaluate the current evidence regarding the impact of continuous positive airway pressure therapy on CAD outcomes in OSA patients.
\end{abstract}

Keywords: Obstructive sleep apnoea (OSA); intermittent hypoxia; coronary artery disease (CAD); cardiovascular disease; continuous positive airway pressure

Submitted Jul 26, 2018. Accepted for publication Dec 13, 2018.

doi: $10.21037 /$ jtd.2018.12.75

View this article at: http://dx.doi.org/10.21037/jtd.2018.12.75

\section{Introduction}

Obstructive sleep apnoea (OSA) has many negative potential consequences for the individual patient, including impaired daytime functioning and reduced quality of life, with the primary aim of treatment often to achieve a reduction in symptom burden (1). At a societal level, however, the most important consequence of OSA may be its association with an increased risk of cardiovascular disease, and of cardiovascular death. A substantial body of evidence from in vitro, animal, clinical, and epidemiological studies now suggests that OSA may have a direct negative effect on cardiovascular outcomes, independent of shared co-morbidities such as obesity and type 2 diabetes (T2D) (2). OSA appears to confer an increased risk of a number of cardiovascular diseases, including hypertension, arrhythmia, stroke, and heart failure. However, in this review we will focus on the relationship of OSA with coronary artery disease (CAD), and will discuss how OSA may contribute to the development of CAD, how OSA relates to CAD incidence and outcomes, and what effect treating OSA may have on patients with CAD.

\section{Why might OSA promote CAD?}

A full discussion of the mechanisms by which OSA may lead to the development of atherosclerosis and subsequent clinically overt CAD are beyond the scope of this review and is covered in detail elsewhere in this issue of the Fournal of Thoracic Disease. In brief, OSA appears to promote cardiovascular disease via a number of pathobiological triggers, including intermittent hypoxia (IH), sleep fragmentation, and intra-thoracic pressure swings leading to altered cardiac and pulmonary vascular haemodynamics (3). Of these, $\mathrm{IH} —$ caused by the repetitive cycles of desaturation and reoxygenation that characterise OSA-may play the most important role. IH leads to a number of maladaptive responses that can ultimately lead to atherosclerosis. First, atherosclerosis is now known to be a chronic inflammatory disease (4), 
and IH appears to promote systemic inflammation, at least partially via activation of the transcription factor NF- $\mathrm{B}$ (5). Data from animal studies would suggest that IH-related inflammation is a critical mediator in the formation of pre-atherosclerotic lesions (2). Second, IH is a potent generator of sympathetic excitation, which in turn promotes endothelial dysfunction and ultimately hypertension and atherosclerosis (2). Third, IH may also have an effect on metabolic health, leading to adipose tissue dysfunction and potential increased risk of T2D (6). Finally, IH appears to lead to the generation of reactive oxygen species, and hence oxidative stress, ultimately leading to endothelial dysfunction and atherosclerosis (7). As we shall discuss in a little more detail later, the effects of $\mathrm{IH}$ are not uniformly negative, with some evidence suggesting a protective effect on the heart via ischaemic preconditioning and the development of coronary collateral vessels (8). Nonetheless, important recently published data emphasise the potentially deleterious effects of $\mathrm{IH}$, and support its primacy over sleep disturbance in causing cardiovascular disease in OSA. In patients receiving CPAP therapy for known OSA, CPAP withdrawal led to elevation in morning blood pressure, an effect that was substantially abrogated by the addition of supplemental nocturnal oxygen therapy (9).

\section{Prevalence and incidence of CAD in OSA cohorts}

Studies from Australia and Europe in the early and mid1990s were among the first to describe an increased prevalence of sleep disordered breathing in subjects with symptomatic CAD. Hung et al. evaluated 101 subjects hospitalized with acute myocardial infarction, comparing them with 53 asymptomatic controls, finding a mean apnoea index of 6.9 events/hour in the former versus 1.4 events/hour among controls (10). A similar study from Sweden enrolled 142 men undergoing evaluation of suspected CAD, along with 50 age-matched volunteers; $6 \%$ of the control subjects had OSA [defined as an apnoea-hypopnoea index (AHI) $\geq 15$ events/hour], compared with $24 \%$ of those in the symptomatic group (11). Another Swedish study, comparing 62 patients requiring intensive care admission with unstable angina or myocardial infarction with age, sex, and BMI-matched controls, again found a higher prevalence of OSA (defined as a respiratory disturbance index $\geq 10$ events/hour) in the cardiac patients (30.7\% versus $13 \%$ ) (12). A cross-sectional analysis of 6,424 individuals enrolled in the North American community-based Sleep Heart Health Study examined the other side of this coin, finding that OSA predicted prevalent CAD. The burden of self-reported CAD among these subjects increased from $9 \%$ in the lowest $\mathrm{AHI}$ quartile to $19 \%$ in the highest, a relationship that survived adjustment for confounding variables (adjusted OR for CAD in highest AHI quartile 1.27 ; 95\% CI: 0.99-1.62) (13).

Data from hospital clinic based studies have suggested that patients with OSA are more likely to go on to develop CAD. Among 182 Swedish men attending a sleep service, who were free of all significant cardio-metabolic co-morbidities at baseline, inadequately treated OSA was associated with an increased likelihood of subsequent symptomatic CAD (adjusted OR 5.4) over a seven year follow up period (14). A subsequent extended analysis by the same authors, which included patients with hypertension and diabetes mellitus, found a CAD incidence of $16.2 \%$ in subjects with OSA, compared to $5.4 \%$ in those without. Adjustment for confounding variables in a Poisson regression model showed OSA at enrollment to be associated with a HR of 4.50 (95\% CI: 1.83-11.6) for incident CAD (15). These findings were supported by a large, single centre Spanish observational study, which compared long term cardiovascular outcomes between patients with varying degrees of sleep disordered breathing and healthy, non-apnoeic subjects (16). In a study cohort of 1,651 individuals, untreated severe OSA was associated with a markedly increased likelihood of fatal (OR 2.87; 95\% CI: 1.17-7.51) and non-fatal (OR 3.17; 95\% CI: 1.12-7.51) cardiovascular disease.

More modest relationships between OSA and CAD are suggested by community based studies. In a longitudinal analysis of 4,422 (56.4\% female) subjects enrolled in the Sleep Heart Health Study who were followed for a median of 8.7 years, severe OSA predicted an increased risk of developing symptomatic CAD, but only in men aged 70 or younger (HR 1.68; 95\% CI: 1.02-2.76) (17). However, another analysis of this cohort has shown severe OSA to be an independent predictor of death, and in particular death related to CAD (18). While this relationship was again strongest in men under 70 years of age (adjusted HR 2.09; 95\% CI: 1.31-3.33), it was nonetheless seen across the entire study population (adjusted HR 1.46; 95\% CI: 1.14-1.86). Participants in the other sentinel communitybased prospective observational study of OSA, the Wisconsin Sleep Cohort, were significantly younger than those in the Sleep Heart Health Study. This may provide at least a partial explanation for the much stronger association seen between OSA and CAD in the former, wherein an AHI of $\geq 30$ conferred a greater than two fold risk of incident 
CAD (adjusted HR 2.63; 95\% CI: 1.13-6.10) over a period of approximately 18,000 person years (19).

The inevitable meta-analyses suggest that OSA confers an increased risk of incident clinically overt CAD in men, with an apparent weaker relationship between OSA and CAD in women (20,21). A systematic review of untreated OSA and long-term adverse outcomes suggested that any negative effect of significant OSA on cardiovascular events was attenuated by female gender, age, a lack of daytime sleepiness, and obesity (22). Overall, there is relatively strong, but not uniform, evidence from clinical and population studies to support an important role for OSA in promoting the evolution of $\mathrm{CAD}$, particularly in younger male patients.

\section{Imaging studies of the coronary vasculature in OSA}

Studies of the coronary vasculature have generally suggested that OSA patients carry a greater subclinical CAD burden than non-apnoeic individuals. These studies can be broadly divided into those using invasive and non-invasive imaging methods, and those directly and indirectly assessing plaque burden in the coronary arteries.

A relatively small number of studies have used invasive techniques: for example, a study of 59 consecutive Japanese patients undergoing coronary angiography following myocardial infarction or for investigation of angina pectoris evaluated the relationship of nocturnal hypoxaemia with coronary atherosclerosis, as measured by the Gensini score (23). Of these patients, $72.9 \%$ had sleep disordered breathing, as defined by an ODI $>5$. ODI correlated significantly with increased Gensini scores $(\mathrm{r}=0.45)$, and the mean Gensini score was higher in subjects with an ODI $\geq 15$ (37.2 \pm 22.7 vs. $21.3 \pm 17.3)$. Similarly, the mean plaque volume was higher in subjects with OSA than those without in a Canadian study of 19 subjects with stable CAD undergoing coronary intravascular ultrasound (IVUS) studies $\left(238 \pm 69\right.$ vs. $\left.169 \pm 64 \mathrm{~mm}^{3}\right)$ (24), findings supported by a larger study of Singaporean patients undergoing clinically indicated coronary angiography (25). In this latter study, patients underwent coronary IVUS and a home cardiorespiratory sleep study; among the 93 subjects who completed the study, a significantly greater plaque volume was seen in patients with moderate-severe OSA when compared with those with an $\mathrm{AHI}<15$, an association that remained significant after adjustment for confounding factors such as obesity and smoking history.
Invasive angiography is obviously resource intensive, and carries small but significant risks of complications, limiting the size and nature of the study populations in which it can be justifiably used. Measurement of coronary artery calcification (CAC) by cardiac CT allows the non-invasive estimation of coronary artery plaque volume, and has been shown to predict coronary and other cardiovascular events, beyond conventional risk models (26). A large retrospective study of 202 subjects with suspected CAD who underwent PSG within three years of cardiac CT being performed for clinically indicated evaluation of CAC (27). CAC was identified in $67 \%$ of OSA patients and $31 \%$ of those without OSA, the median CAC score was higher in the most severe AHI quartile than the lowest, and multivariate regression analysis revealed severe sleep disordered breathing to be an independent predictor of CAC (adjusted OR 3.30; 95\% CI: 1.20-9.40).

The studies discussed above are limited by their reliance on subjects presenting with suspected CAD and by the high prevalence of important risk factors for atherogenesis in their study cohorts. However, similar relationships have been observed in studies of individuals without clinically apparent CAD. The Heinz Nixdorf Recall study was a German community based observational study estimating the predictive utility of CAC scoring in predicting subsequent cardiovascular events in subjects free of overt CAD. A large subgroup of these subjects $(n=1,604)$ underwent home cardiorespiratory sleep studies in parallel with their cardiac imaging; OSA was highly prevalent in this cohort $(22 \%$ had an $\mathrm{AHI} \geq 15)$ and was independently related to the amount of CAC seen in men aged $\leq 65$ years and in women of any age (28). The MultiEthnic Study of Atherosclerosis (MESA) used a similar design in 1,465 North American community based subjects, again without known cardiovascular disease, but using home PSG and actigraphy to give a more complete picture of the participants' sleep. The prevalence of CAC within this cohort was independently predicted by an $\mathrm{AHI} \geq 30$, but also seemed to be related to sleep fragmentation and reduced proportions of $\mathrm{N} 3$ sleep, perhaps suggesting a separate role for impaired sleep quality in promoting CAC development (29).

Formal coronary CT angiography may have some advantages over CAC scoring, insofar as it also allows quantification of 'soft', non-calcified plaque burden. We evaluated sub-clinical coronary atherosclerosis in a cohort of 29 otherwise healthy non-smoking men free of cardiometabolic disease undergoing inpatient sleep studies for suspected OSA, and found that AHI was independently 
correlated with coronary plaque volume, and that greater plaque volumes were seen in patients with moderatesevere OSA when compared with those with no-mild sleep disordered breathing (30), independent of metabolic health.

A number of investigators have estimated coronary atherosclerosis by assessing instead its surrogate—carotid artery intima media thickness (IMT). Carotid IMT measured by ultrasonography correlates well with anatomic findings, and further is a well-validated marker of coronary atherosclerosis, as well as a predictor of cardiovascular morbidity and mortality (31). Baguet et al. evaluated carotid IMT in 82 newly diagnosed OSA patients, who were largely free of antecedentally diagnosed cardiovascular or metabolic disease (32). Mean nocturnal $\mathrm{SpO}_{2}$ correlated inversely with carotid IMT measurements $(r=-0.30$, $\mathrm{P}=0.006$ ), with multivariate logistic regression analysis showing it to be an independent predictor of carotid artery hypertrophy (adjusted OR 3.9; 95\% CI: 1.1-12.7). Carotid IMT was higher in OSA subjects in a study of 52 Japanese men free of co-morbidities, increasing from $0.71 \pm 0.07 \mathrm{~mm}$ in controls to $1.07 \pm 0.03 \mathrm{~mm}$ in those with an $\mathrm{AHI}>5$ (33). Patients with moderate-severe OSA had higher carotid IMT measurements than those with mild disease $(1.16 \pm 0.07$ vs. $0.92 \pm 0.07 \mathrm{~mm}$ ). A Brazilian study of 15 subjects with mild-moderate OSA, 15 subjects with severe OSA, and 12 age-, sex-, and BMI-matched controls rigorously screened participants for occult diabetes or hypertension, including only subjects free of co-morbid disease (34). Among these patients, carotid IMT was greater in those with severe OSA than those with milder disease or controls, and correlated significantly with increasing AHI $(r=0.44, \mathrm{P}=0.004)$. These investigators went on to randomise 24 otherwise healthy patients with severe sleep disordered breathing to either CPAP or no treatment for 4 months (35). Carotid IMT decreased significantly in the CPAP group $(\Delta-62 \mathrm{~mm}$; $95 \%$ CI: -110 to $-15 \mathrm{~mm}$ ), but not in the untreated group ( $\Delta 8 \mathrm{~mm}$; 95\% CI: -21 to $37 \mathrm{~mm}$ ). These findings seem to be independent of the effects of any undiagnosed hypertension, with a similar study from the same group suggesting that OSA and hypertension have an additive effect on carotid IMT (36).

All of the above data regarding carotid IMT are derived from sleep laboratory cohorts with some healthy volunteers. A cross sectional analysis of 985 participants in the community based Sleep Heart Health Study, excluding all subjects with known CAD, but including a large proportion of smokers and individuals with hypertension and T2D, compared carotid IMT across AHI quartiles (37). In unadjusted analysis, carotid IMT — and the likelihood of finding carotid artery plaque-increased with increasing OSA severity, but this relationship disappeared when confounding variables were included in their statistical models. Conversely, longitudinal analyses from a subset of subjects in the Wisconsin Sleep Cohort found that baseline $\mathrm{AHI}$ and nadir nocturnal $\mathrm{SpO}_{2}$ had a statistically significant (if clinically uncertain) relationship with the development of the ensuing 13 years $(38,39)$.

These data-along with data from animal models suggesting that carotid artery hypertrophy could be driven by simple snoring rather than more florid OSA (40) mean that the relationship of carotid IMT with OSA is not an unequivocal one. Furthermore, it is unclear if carotid ultrasonography is as representative of coronary atherosclerosis in OSA patients as in non-apnoeic populations: a comparison of coronary CT angiography and carotid IMT measurement in 33 patients with OSA found IMT was specific, but insensitive in the detection of subclinical CAD (41).

In summary, a reasonable, but not unequivocal, body of evidence suggests that OSA is associated with an increased burden of coronary atherosclerosis in patients presenting with symptomatic CAD, as well as in sleep laboratory cohorts, and perhaps in community based population studies. Little is known about the effect of CPAP therapy on coronary imaging findings, something which may provide a viable surrogate endpoint for primary prevention studies of CAD in OSA patients.

\section{Relationships of OSA with outcomes in CAD patients}

OSA seems likely to predispose individuals to the development of CAD, but does it influence outcomes in patients presenting with acute coronary syndromes (ACS) or requiring urgent care for symptomatic CAD? Much of the data discussed above, along with the observation that OSA seems to be associated with an increased risk of nocturnal sudden cardiac death (42), might suggest that it exerts a significant negative effect. However, in recent years interest has grown on the potential protective effect that OSA and associated IH may have in cases of myocardial infarction, via the phenomenon of ischaemic preconditioning. Ischaemic preconditioning may occur in the myocardium due to repeated exposure to $\mathrm{IH}$ occurring over a prolonged period of time below the threshold required to cause significant injury, leading to the upregulation of adaptive pathways 
which may ultimately facilitate myocardial survival during prolonged acute tissue hypoxia (8). There is some evidence to suggest that this may occur in OSA. First, the negative effect of OSA on cardiovascular morbidity and mortality seems much less pronounced in older populations, where the diagnosis may even be associated with a survival advantage (8). Second, individuals with OSA seem more likely have developed a collateral coronary circulation than those without $(43,44)$. Finally, two single centre studies have found that peak cardiac troponin levels (a surrogate marker for infarct size) during presentations with ACS are lower in patients with significant sleep disordered breathing than those without $(45,46)$.

There is, however, evidence to the contrary, suggesting that increasing OSA severity leads to increased infarct size. The largest study examining the relationship of OSA with troponin release during ACS found an elevated peak plasma troponin concentration in patients with the highest AHI (47). This was a multicentre study involving a significantly greater number of patients than the studies mentioned above. The gold standard investigation for determining infarct size post-ACS is cardiac magnetic resonance imaging (CMR). A study of 56 German patients with acute myocardial infarction who underwent percutaneous intervention (PCI) found that an $\mathrm{AHI} \geq 15$ was associated with a larger infarct size, reduced myocardial salvage, and a lower left ventricular ejection fraction on CMR at 3 months post PCI (48).

Whatever about its effect on acute ACS outcomes, untreated OSA appears to worsen prognosis in the mediumlong term following requirement for PCI. The largest study to date prospectively examining outcomes in patients with OSA who have undergone PCI is the Sleep and Stent Study, which involved eight centres in Asia and Brazil (49). This study observed outcomes in 1,311 patients who had a cardiorespiratory sleep study within seven days of successful PCI; $45.3 \%$ of patients had an AHI $\geq 15$, which after a median follow up of 1.9 years conferred a $57 \%$ increase in relative risk of a major adverse cardiac or cerebrovascular event. Similar results have been reported from smaller single centre studies (50-54) and a recent meta-analysis of the published data (55).

Consequently, one might anticipate that CPAP treatment might serve as potential primary prevention strategy in OSA patients at risk of $\mathrm{CAD}$, or as secondary prevention in those with established disease. As we shall discuss in the final section of this review, however, the clinical trials performed to date have not necessarily produced the expected results.

\section{Does treating OSA modify CAD outcomes?}

Conducting randomised controlled trials of the impact of CPAP therapy on hard cardiovascular outcomes poses a number of very significant logistical and ethical difficulties. First, in order to accrue sufficient patient years to achieve enough power to identify any benefit from CPAP as a primary or secondary prevention intervention, study cohorts much be large, and must be followed for a relatively prolonged period of time. Second, even if CPAP turns out be of no benefit in reducing CAD, it certainly is of symptomatic benefit for many patients, and depriving some of these patients of treatment for months or years due to enrolment in the placebo arm of a RCT is ethically questionable. Finally, although CPAP is a highly effective therapy when used, a substantial proportion of patients struggle to use it, with adherence to therapy notoriously variable.

Perhaps as a consequence of these difficulties, it is only in the recent past that evidence has been published from RCTs examining the effect of CPAP on major adverse cardiovascular events. Prior this, we had quite reassuring data from a significant number of well-designed and conducted RCTs demonstrating the ability of CPAP to (modestly) lower blood pressure (56), which gave hope that this would in turn lead to a long-term reduction in cardiovascular morbidity and mortality. We also had the results of a number of single centre, often retrospective, unblinded studies of CAD disease outcomes which suggested that CPAP may be of use as both a primary and secondary prevention measure, including recent data from Sweden, which suggest similar medium term risk of cardiovascular events in patients with established CAD receiving CPAP compared to non-apnoeic controls (57-60).

One of the most notable of these was a large Spanish study, which investigated cardiovascular outcomes in a large cohort of five different groups (I) healthy men ( $\mathrm{n}=264)$, (II) simple snorers $(\mathrm{n}=377)$, (III) untreated mild-moderate OSA ( $\mathrm{n}=403$ ), (IV) severe untreated OSA (mean AHI 43 vents/hour, $\mathrm{n}=235)$, and $(\mathrm{V})$ those with OSA and treated with CPAP (mean AHI 42 events/hour, $n=372$ ), with a mean follow up period of 10 years (16). As mentioned previously, patients with severe, untreated OSA in this population had a markedly increased risk of cardiovascular events and death. However, event rates in OSA patients using CPAP therapy were essentially identical to healthy patients and simple snorers, potentially suggesting a rather dramatic modifying effect from CPAP on cardiovascular outcomes. This was 
a well conducted study amongst five distinct diagnostic groups with a long follow up period, with similar AHI, BMI and age in the severe OSA groups (treated and untreated), thus comparison of the incidence of cardiovascular events appeared valid. However, it was limited as an observational study, and it is important to note that those that had untreated OSA may have been a self-selecting cohort of patients who refused treatment, and potentially had other negative health behaviours.

In the recent past, a number of investigators have managed to overcome some of the obstacles to conducting prospective, randomised trials in this area, and we now have data from two large RCTs investigating the impact of CPAP treatment on cardiovascular outcomes in patients with OSA and established cardiovascular disease. The SAVE (Sleep Apnea Cardiovascular Endpoints) trial was a large international study that investigated the impact of CPAP on mortality or hospitalisation caused by cardiovascular events (primary outcomes) in non-sleepy OSA patients with known cardiovascular disease (61). Secondary outcomes sought to determine differences in other cardiovascular composite end points, health related quality of life, OSA symptoms, and mood. Over 2,000 patients completed the trial, and there was no significant difference CPAP and usual care in terms of primary outcomes: 229 patients met the primary end point in the CPAP group and 207 in the usual care group. Additionally, no differences in outcomes were found even when adjusting for severity of OSA, body mass index or presence of metabolic disorders. Interestingly, although these patients were ostensibly non-sleepy, Epworth scores were reduced by CPAP, and quality of life measures improved.

The RICCADSA (randomised intervention with continuous positive airway pressure in CAD and OSA) trial investigated the impact of CPAP in asymptomatic OSA $($ ESS $<10)$ on cardiovascular outcomes in patients who had angiographically confirmed CAD (and had undergone treatment for their CAD) (62). Subjects were either randomised to CPAP or usual care. The primary outcome was composite: repeat revascularisation, MI, stroke and mortality due to cardiovascular disease. The groups were well matched. There was no significant difference between the two groups in the incidence of the composite outcomes [CPAP group 4.18, (95\% CI: 2.75-6.35) vs. no CPAP group 5.21 (95\% CI: 3.57-7.60), $\mathrm{P}=0.449$ ] per 100 person years. Additionally, there were no significant differences between the two groups in the individual cardiovascular outcomes. Diabetes and previous revascularisation, but not OSA, were predictive of adverse outcomes.

These results caused surprise (and perhaps a degree of consternation) in the sleep research community, and a number of limitations of the studies were raised. First, the average AHI in both studies was 29 events/hour, consistent with moderate OSA. Second, the mean CPAP usage was less than 4 hours, which may have left a significant degree of OSA untreated in these individuals. Specifically, this may have left a significant degree or REM-OSA untreated, which seems to have a particularly negative effect on cardiometabolic health (63). Notably, if a cut off level of 4 hours of CPAP use was applied for adherence in the RICCADSA trial, a significant difference was noted in the incidence of outcomes (CPAP >4 hours 2.31 (95\% CI: 0.96-5.54) vs. CPAP <4 hours 5.32 (95\% CI: 3.96-7.15) per 100 personyears, significantly different to no CPAP (HR 0.29; $95 \%$ CI: $0.1-0.86 ; \mathrm{P}=0.026$ ). It would appear that the absence of subjective daytime sleepiness significantly reduces the likelihood of OSA patients with CAD being adherent to CPAP therapy (64). Third, the patients were not subjectively sleepy, which may have had a limiting effect on adherence, but which also raised the question of whether sleepy OSA patients have different outcomes than their non-sleepy counterparts.

These were secondary prevention trials, and it may be the case that even excellent adherence to treatment of very severe OSA may be of limited benefit in preventing further cardiac events in the presence of well-established CAD. There is a paucity of RCT data examining the utility of $\mathrm{CPAP}$ as a primary prevention measure. One study, which attempted to do just this, enrolled 723 non-sleepy patients, free of clinically overt CAD, with at least moderately severe OSA across several Spanish sleep centres (65). Subjects were randomised to either CPAP therapy or usual care, and followed for a median of 4 years. No significant difference was seen between groups in rates of major cardiovascular events, although a post hoc analysis suggested a relative risk reduction of $28 \%$ in patients who were adherent to CPAP therapy. This study neatly demonstrates a number of the difficulties involved in conducting a primary prevention trial using hard cardiovascular endpoints: despite being a well run, nicely designed RCT conducted by experienced investigators in a relatively large population followed for a significant period of time, the trial was likely underpowered, and the patients likely under-treated.

Overall we are left with as many questions as answers when it comes to OSA treatment and cardiovascular outcomes. Perhaps the selection of the right cohort of 
individuals is key: for example, in the RICCADSA trial, had all the patients been adherent to CPAP for a minimum of 4 hours per night would a difference have been seen? Perhaps the severe end of the OSA spectrum is where CPAP treatment is crucial rather than mild-moderate OSA? Are other established and emerging treatment modalities likely to be more effective than CPAP in improving cardiovascular outcomes? It is clear that further randomised controlled trials with carefully phenotyped cohorts are required to answer the question of whether treating OSA is important in reducing cardiovascular risk.

\section{Acknowledgements}

None.

\section{Footnote}

Conflict of Interest: The authors have no conflicts of interest to declare.

\section{References}

1. Garvey JF, Pengo MF, Drakatos P, et al. Epidemiological aspects of obstructive sleep apnea. $\mathrm{J}$ Thorac Dis 2015;7:920-9.

2. Lévy P, Ryan S, Oldenburg O, et al. Sleep apnoea and the heart. Eur Respir Rev 2013;22:333-52.

3. Kent BD, Ryan S, McNicholas WT. Obstructive sleep apnea and inflammation: relationship to cardiovascular comorbidity. Respir Physiol Neurobiol 2011;178:475-81.

4. Ross R: Atherosclerosis--an inflammatory disease. N Engl J Med 1999;340:115-26.

5. Ryan S, Taylor CT, McNicholas WT. Selective activation of inflammatory pathways by intermittent hypoxia in obstructive sleep apnea syndrome. Circulation 2005;112:2660-7.

6. Kent BD, McNicholas WT, Ryan S. Insulin resistance, glucose intolerance and diabetes mellitus in obstructive sleep apnoea. J Thorac Dis 2015;7:1343-57.

7. Lavie L, Lavie P. Molecular mechanisms of cardiovascular disease in OSAHS: the oxidative stress link. Eur Respir J 2009;33:1467-84.

8. Aronson D, Lavie L, Lavie P. Does OSA Upregulate Cardioprotective Pathways to an Ischemic Insult? Chest 2018;153:295-7.

9. Turnbull CD, Sen D, Kohler M, et al. Effect of Supplemental Oxygen on Blood Pressure in Obstructive
Sleep Apnea (SOX): A Randomised, CPAP Withdrawal Trial. Am J Respir Crit Care Med 2018. [Epub ahead of print].

10. Hung J, Whitford EG, Parsons RW, et al. Association of sleep apnoea with myocardial infarction in men. Lancet 1990;336:261-4.

11. Mooe T, Rabben T, Wiklund U, et al. Sleep-disordered breathing in men with coronary artery disease. Chest 1996;109:659-63.

12. Peker Y, Kraiczi H, Hedner J, et al. An independent association between obstructive sleep apnoea and coronary artery disease. Eur Respir J 1999;14:179-84.

13. Shahar E, Whitney CW, Redline S, et al. Sleep-disordered breathing and cardiovascular disease: cross-sectional results of the Sleep Heart Health Study. Am J Respir Crit Care Med 2001;163:19-25.

14. Peker Y, Hedner J, Norum J, et al. Increased incidence of cardiovascular disease in middle-aged men with obstructive sleep apnea: a 7-year follow-up. Am J Respir Crit Care Med 2002;166:159-65.

15. Peker Y, Carlson J, Hedner J. Increased incidence of coronary artery disease in sleep apnoea: a long-term follow-up. Eur Respir J 2006;28:596-602.

16. Marin JM, Carrizo SJ, Vicente E, et al.: Long-term cardiovascular outcomes in men with obstructive sleep apnoea-hypopnoea with or without treatment with continuous positive airway pressure: an observational study. Lancet 2005;365:1046-53.

17. Gottlieb DJ, Yenokyan G, Newman AB, et al. Prospective study of obstructive sleep apnea and incident coronary heart disease and heart failure: the sleep heart health study. Circulation 2010;122:352-60.

18. Punjabi NM, Caffo BS, Goodwin JL, et al. Sleepdisordered breathing and mortality: a prospective cohort study. PLoS medicine 2009;6:e1000132.

19. Hla KM, Young T, Hagen EW, et al. Coronary heart disease incidence in sleep disordered breathing: the Wisconsin Sleep Cohort Study. Sleep 2015;38:677-84.

20. Dong JY, Zhang YH, Qin LQ. Obstructive sleep apnea and cardiovascular risk: meta-analysis of prospective cohort studies. Atherosclerosis 2013;229:489-95.

21. Loke YK, Brown JW, Kwok CS, et al. Association of obstructive sleep apnea with risk of serious cardiovascular events: a systematic review and meta-analysis. Circ Cardiovasc Qual Outcomes 2012;5:720-8.

22. Kendzerska T, Mollayeva T, Gershon AS, et al. Untreated obstructive sleep apnea and the risk for serious long-term adverse outcomes: a systematic review. Sleep Med Rev 
2014;18:49-59.

23. Hayashi M, Fujimoto K, Urushibata K, et al. Nocturnal oxygen desaturation correlates with the severity of coronary atherosclerosis in coronary artery disease. Chest 2003;124:936-41.

24. Turmel J, Series F, Boulet LP, et al. Relationship between atherosclerosis and the sleep apnea syndrome: an intravascular ultrasound study. Int J Cardiol 2009;132:203-9.

25. Tan A, Hau W, Ho HH, et al. OSA and coronary plaque characteristics. Chest 2014;145:322-30.

26. Erbel R, Mohlenkamp S, Moebus S, et al. Coronary risk stratification, discrimination, and reclassification improvement based on quantification of subclinical coronary atherosclerosis: the Heinz Nixdorf Recall study. J Am Coll Cardiol 2010;56:1397-406.

27. Sorajja D, Gami AS, Somers VK, et al. Independent association between obstructive sleep apnea and subclinical coronary artery disease. Chest 2008;133:927-33.

28. Weinreich G, Wessendorf TE, Erdmann T, et al. Association of obstructive sleep apnoea with subclinical coronary atherosclerosis. Atherosclerosis 2013;231:191-7.

29. Lutsey PL, McClelland RL, Duprez D, et al. Objectively measured sleep characteristics and prevalence of coronary artery calcification: the Multi-Ethnic Study of Atherosclerosis Sleep study. Thorax 2015;70:880-7.

30. Kent BD, Garvey JF, Ryan S, et al. Severity of obstructive sleep apnoea predicts coronary artery plaque burden: a coronary computed tomographic angiography study. Eur Respir J 2013;42:1263-70.

31. Lorenz MW, Markus HS, Bots ML, et al. Prediction of clinical cardiovascular events with carotid intimamedia thickness: a systematic review and meta-analysis. Circulation 2007;115:459-67.

32. Baguet JP, Hammer L, Levy P, et al. The severity of oxygen desaturation is predictive of carotid wall thickening and plaque occurrence. Chest 2005;128:3407-12.

33. Minoguchi K, Yokoe T, Tazaki T, et al. Increased carotid intima-media thickness and serum inflammatory markers in obstructive sleep apnea. Am J Respir Crit Care Med 2005;172:625-30.

34. Drager LF, Bortolotto LA, Lorenzi MC, et al. Early signs of atherosclerosis in obstructive sleep apnea. Am J Respir Crit Care Med 2005;172:613-8.

35. Drager LF, Bortolotto LA, Figueiredo AC, et al. Effects of continuous positive airway pressure on early signs of atherosclerosis in obstructive sleep apnea. Am J Respir Crit Care Med 2007;176:706-12.
36. Drager LF, Bortolotto LA, Krieger EM, et al. Additive effects of obstructive sleep apnea and hypertension on early markers of carotid atherosclerosis. Hypertension 2009;53:64-9.

37. Wattanakit K, Boland L, Punjabi NM, et al. Relation of sleep-disordered breathing to carotid plaque and intimamedia thickness. Atherosclerosis 2008;197:125-31.

38. Gunnarsson SI, Peppard PE, Korcarz CE, et al. Obstructive sleep apnea is associated with future subclinical carotid artery disease: thirteen-year follow-up from the Wisconsin sleep cohort. Arterioscler Thromb Vasc Biol 2014;34:2338-42.

39. Gunnarsson SI, Peppard PE, Korcarz CE, et al. Minimal nocturnal oxygen saturation predicts future subclinical carotid atherosclerosis: the Wisconsin sleep cohort. J Sleep Res 2015;24:680-6.

40. Cho JG, Witting PK, Verma M, et al. Tissue vibration induces carotid artery endothelial dysfunction: a mechanism linking snoring and carotid atherosclerosis? Sleep 2011;34:751-7.

41. Murphy AM, Thomas A, Crinion SJ, et al. Intermittent hypoxia in obstructive sleep apnoea mediates insulin resistance through adipose tissue inflammation. Eur Respir J 2017;49.

42. Gami AS, Howard DE, Olson EJ, et al. Day-night pattern of sudden death in obstructive sleep apnea. $\mathrm{N}$ Engl J Med 2005;352:1206-14.

43. Steiner S, Schueller PO, Schulze V, et al. Occurrence of coronary collateral vessels in patients with sleep apnea and total coronary occlusion. Chest 2010;137:516-20.

44. Ben Ahmed H, Boussaid H, Longo S, et al. Impact of obstructive sleep apnea in recruitment of coronary collaterality during inaugural acute myocardial infarction. Ann Cardiol Angeiol (Paris) 2015;64:273-8.

45. Shah N, Redline S, Yaggi HK, et al. Obstructive sleep apnea and acute myocardial infarction severity: ischemic preconditioning? Sleep Breath 2013;17:819-26.

46. Sánchez-de-la-Torre A, Soler X, Barbe F, et al. Cardiac Troponin Values in Patients With Acute Coronary Syndrome and Sleep Apnea: A Pilot Study. Chest 2018;153:329-38.

47. Barbé F, Sanchez-de-la-Torre A, Abad J, et al. Effect of obstructive sleep apnoea on severity and short-term prognosis of acute coronary syndrome. Eur Respir J 2015;45:419-27.

48. Buchner S, Satzl A, Debl K, et al. Impact of sleepdisordered breathing on myocardial salvage and infarct size in patients with acute myocardial infarction. Eur Heart J 
2014;35:192-9.

49. Lee CH, Sethi R, Li R, et al. Obstructive Sleep Apnea and Cardiovascular Events After Percutaneous Coronary Intervention. Circulation 2016;133:2008-17.

50. Peker Y, Hedner J, Kraiczi H, et al. Respiratory disturbance index: an independent predictor of mortality in coronary artery disease. Am J Respir Crit Care Med 2000;162:81-6.

51. Yumino D, Tsurumi Y, Takagi A, et al. Impact of obstructive sleep apnea on clinical and angiographic outcomes following percutaneous coronary intervention in patients with acute coronary syndrome. Am J Cardiol 2007;99:26-30.

52. Xie J, Sert Kuniyoshi FH, Covassin N, et al. Nocturnal Hypoxemia Due to Obstructive Sleep Apnea Is an Independent Predictor of Poor Prognosis After Myocardial Infarction. J Am Heart Assoc 2016;5(8).

53. Mazaki T, Kasai T, Yokoi H, K et al. Impact of SleepDisordered Breathing on Long-Term Outcomes in Patients With Acute Coronary Syndrome Who Have Undergone Primary Percutaneous Coronary Intervention. J Am Heart Assoc 2016;5(6).

54. Jia S, Zhou YJ, Yu Y, et al. Obstructive sleep apnea is associated with severity and long-term prognosis of acute coronary syndrome. J Geriatr Cardiol 2018;15:146-52.

55. Qu H, Guo M, Zhang Y, et al. Obstructive sleep apnea increases the risk of cardiac events after percutaneous coronary intervention: a meta-analysis of prospective cohort studies. Sleep Breath 2018;22:33-40.

56. Bratton DJ, Gaisl T, Wons AM, et al. CPAP vs Mandibular Advancement Devices and Blood Pressure in Patients With Obstructive Sleep Apnea: A Systematic Review and Meta-analysis. JAMA 2015;314:2280-93.

57. Doherty LS, Kiely JL, Swan V, et al. Long-term effects

Cite this article as: Mandal S, Kent BD. Obstructive sleep apnoea and coronary artery disease. J Thorac Dis 2018;10(Suppl 34):S4212-S4220. doi: 10.21037/jtd.2018.12.75 of nasal continuous positive airway pressure therapy on cardiovascular outcomes in sleep apnea syndrome. Chest 2005;127:2076-84.

58. Cassar A, Morgenthaler TI, Lennon RJ, et al. Treatment of obstructive sleep apnea is associated with decreased cardiac death after percutaneous coronary intervention. J Am Coll Cardiol 2007;50:1310-4.

59. Wu X, Lv S, Yu X, et al. Treatment of OSA reduces the risk of repeat revascularization after percutaneous coronary intervention. Chest 2015;147:708-18.

60. Peker Y, Thunstrom E, Glantz H, et al. Outcomes in coronary artery disease patients with sleepy obstructive sleep apnoea on CPAP. Eur Respir J 2017;50(6).

61. McEvoy RD, Antic NA, Heeley E, et al. CPAP for Prevention of Cardiovascular Events in Obstructive Sleep Apnea. N Engl J Med 2016;375:919-31.

62. Peker Y, Glantz H, Eulenburg C, et al. Effect of Positive Airway Pressure on Cardiovascular Outcomes in Coronary Artery Disease Patients with Nonsleepy Obstructive Sleep Apnea. The RICCADSA Randomized Controlled Trial. Am J Respir Crit Care Med 2016;194:613-20.

63. Mokhlesi B, Finn LA, Hagen EW, et al. Obstructive sleep apnea during REM sleep and hypertension. results of the Wisconsin Sleep Cohort. Am J Respir Crit Care Med 2014;190:1158-67.

64. Luyster FS, Strollo PJ Jr, Thunstrom E, et al. Long-term use of continuous positive airway pressure therapy in coronary artery disease patients with nonsleepy obstructive sleep apnea. Clin Cardiol 2017;40:1297-302.

65. Barbé F, Duran-Cantolla J, Sanchez-de-la-Torre M, et al. Effect of continuous positive airway pressure on the incidence of hypertension and cardiovascular events in nonsleepy patients with obstructive sleep apnea: a randomized controlled trial. JAMA 2012;307:2161-8. 